University of Nebraska - Lincoln

DigitalCommons@University of Nebraska - Lincoln

Faculty Publications from the Department of Electrical \& Computer Engineering, Department Electrical and Computer Engineering

2-15-2007

\title{
Spherical silicon-shell photonic band gap structures fabricated by laser-assisted chemical vapor deposition
}

\author{
H. Wang \\ University of Nebraska-Lincoln \\ Z. Y. Yang \\ University of Nebraska-Lincoln \\ Yongfeng Lu \\ University of Nebraska-Lincoln, ylu2@unl.edu
}

Follow this and additional works at: https://digitalcommons.unl.edu/electricalengineeringfacpub

Part of the Electrical and Computer Engineering Commons

Wang, H.; Yang, Z. Y.; and Lu, Yongfeng, "Spherical silicon-shell photonic band gap structures fabricated by laser-assisted chemical vapor deposition" (2007). Faculty Publications from the Department of Electrical and Computer Engineering. 88.

https://digitalcommons.unl.edu/electricalengineeringfacpub/88

This Article is brought to you for free and open access by the Electrical \& Computer Engineering, Department of at DigitalCommons@University of Nebraska - Lincoln. It has been accepted for inclusion in Faculty Publications from the Department of Electrical and Computer Engineering by an authorized administrator of DigitalCommons@University of Nebraska - Lincoln. 


\title{
Spherical silicon-shell photonic band gap structures fabricated by laser-assisted chemical vapor deposition
}

\author{
H. Wang, Z. Y. Yang, and Y. F. Lu ${ }^{\text {a) }}$ \\ Department of Electrical Engineering, University of Nebraska-Lincoln, Lincoln, Nebraska 68588-0511
}

(Received 23 June 2006; accepted 26 December 2006; published online 15 February 2007)

\begin{abstract}
Laser-assisted chemical vapor deposition was applied in fabricating three-dimensional (3D) spherical-shell photonic band gap (PBG) structures by depositing silicon shells covering silica particles, which had been self-assembled into 3D colloidal crystals. The colloidal crystals of self-assembled silica particles were formed on silicon substrates using the isothermal heating evaporation approach. A continuous wave Nd:YAG laser (1064 nm wavelength) was used to deposit silicon shells by thermally decomposing disilane gas. Periodic silicon-shell/silica-particle PBG structures were obtained. By removing the silica particles enclosed in the silicon shells using hydrofluoric acid, hollow spherical silicon-shell arrays were produced. This technique is capable of fabricating structures with complete photonic band gaps, which is predicted by simulations with the plane wave method. The techniques developed in this study have the potential to flexibly engineer the positions of the PBGs by varying both the silica particle size and the silicon-shell thickness. Ellipsometry was used to investigate the specific photonic band gaps for both structures. (C) 2007 American Institute of Physics. [DOI: 10.1063/1.2472648]
\end{abstract}

\section{INTRODUCTION}

Silicon has been studied as an optical material since the mid-1980s. Recent progresses in microfabrication techniques have been greatly boosting the realization of silicon-based optoelectronic devices. Silicon with microstructures reveals stronger Raman effect, ${ }^{1}$ which leads to the achievement of silicon Raman laser in recent years. ${ }^{2}$ Microstructures in silicon enhance useful quantumlike effects to obtain high optical gains. ${ }^{3}$ Long-range periodical microstructures, i.e., photonic band gap (PBG) structures, have attracted the interests of researchers since $1987 .{ }^{4,5}$ It is imperative to develop simple and efficient methods to fabricate three-dimensional (3D) PBG structures. Among current prevailing techniques for fabricating large-scale 3D PBGs, holographic laser lithography and direct laser writing were intensively studied because these approaches were rapid and flexible. ${ }^{6,7}$ However, these approaches usually use polymers as base materials, which lack high thermal stability and high refractive index. To obtain sufficiently high refractive index contrast against air, which is essential to form complete photonic band gaps, silicon chemical vapor deposition (CVD) is widely used to infiltrate structures with low refractive index. In this study, we developed a method to deposit silicon into colloidal-crystal templates to fabricate 3D photonic band gap structures using laser-assisted chemical vapor deposition (LCVD).

Based on the matured techniques to synthesize ${ }^{8}$ and self-assemble ${ }^{9}$ monodisperse silica particles, silica colloidal crystals are ideal bases for fabricating PBG structures. The colloidal crystals are limited to the face-centered cubic (fcc) structure. Nevertheless, since it possesses the Brillouin zone with the most circular shape, the fcc structure is well accepted as a good candidate to produce large and complete

\footnotetext{
a) Author to whom correspondence should be addressed; electronic mail: ylu2@unl.edu.
}

band gaps. ${ }^{10}$ There are many strategic advantages to use selfassembly of silica particles. First, silica particles can withstand high temperature in CVD processes. Second, the silica nanoparticles with metallic or semiconductor cores can also be self-assembled into high-quality colloidal crystals. It is possible to adjust the optical properties of colloidal crystals by controlling core materials and core-shell geometries. Third, the colloidal crystals are feasible for infiltration of various materials, such as silicon, ${ }^{11,12}$ graphite, ${ }^{13} \mathrm{CdSe},{ }^{14}$ $\mathrm{CdS},{ }^{15}$ and $\mathrm{TiO}_{2} \cdot{ }^{16,17}$ The combinations of core-shell-filling materials provide a wide range of different optical properties.

LCVD is an attractive process for material growth. ${ }^{18} \mathrm{~A}$ variety of materials, such as metals, semiconductors, and insulators can be deposited by LCVD, which can also be applied to the fabrication of 3D PBG structures. Principally, there are two types of LCVD. One is based on direct photochemical decomposition of reactant gases. ${ }^{19}$ The other is based on laser-induced thermal effects. ${ }^{18}$ The latter was used in this study. A laser beam was used to locally create a hot area on the substrate surface where gas decomposition and material deposition occurred. LCVD is a cold-wall process, in which the laser beam only heats up the desirable area instead of the whole chamber or large substrate stage in conventional CVD techniques. Meanwhile, LCVD is a top heating process. The substrates can be heated from the top while they are heated from bottom, so that the substrates remain in a relatively uniform temperature environment. ${ }^{20}$ LCVD is also a fast process. The intensive energy of the laser beam could instantaneously heat substrates to a desired temperature, at which both deposition and annealing can be achieved.

In this study, a fabrication method based on the advantages of both colloidal crystals and LCVD was used. High- 


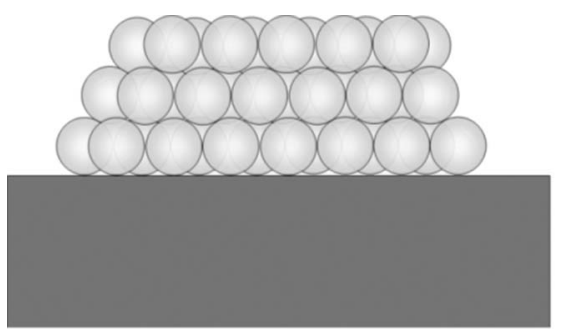

(a)

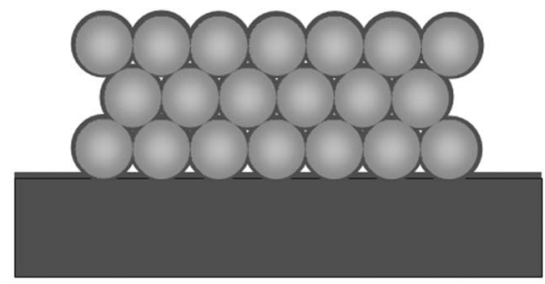

(b)

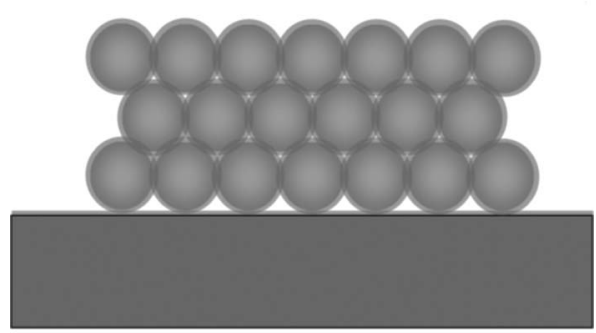

(c)

FIG. 1. LCVD method based on self-assembly of colloidal particles: (a) self-assembly of colloidal silica particles, (b) silica particles with silicon shells deposited in LCVD, and (c) spherical hollow silicon-shell PBG structure.

quality PBG structures were fabricated using this approach. The material and optical properties were characterized and compared with simulation results.

\section{EXPERIMENT DETAILS}

Figure 1 shows the method of fabricating 3D PBG structures and the structures obtained in each step. The PBG structures consisting of colloidal crystals are shown in Fig. 1(a). Self-assembled colloidal crystals are ideal candidates for further silicon infiltration using the LCVD technique. A layer of silicon shell was deposited over the silica particles using the LCVD process [Fig. 1(b)]. The silica/silicon-shell structure could be obtained as the second type of PBG structures in this method. After removing the silica particles with HF acid, hollow spherical silicon-shell arrays can be obtained [Fig. 1(c)]. The silicon shells have a large contrast in the refractive index with air to form a complete photonic band gap. Furthermore, the silicon shells can be used for further processing, such as infiltrating a liquid crystal, whose refractive index is electrically tunable.

The first process step is self-assembly of colloidal particles. The isothermal heating evaporation-induced self-assembly ${ }^{9}$ (IHEISA) was used to assemble colloidal crystals. Suspensions of monodisperse silica particles $(3-5 \mathrm{wt} \%)$ were evaporated in an isothermal environment at $80{ }^{\circ} \mathrm{C}$, leaving colloidal crystals consisting of about 20-30

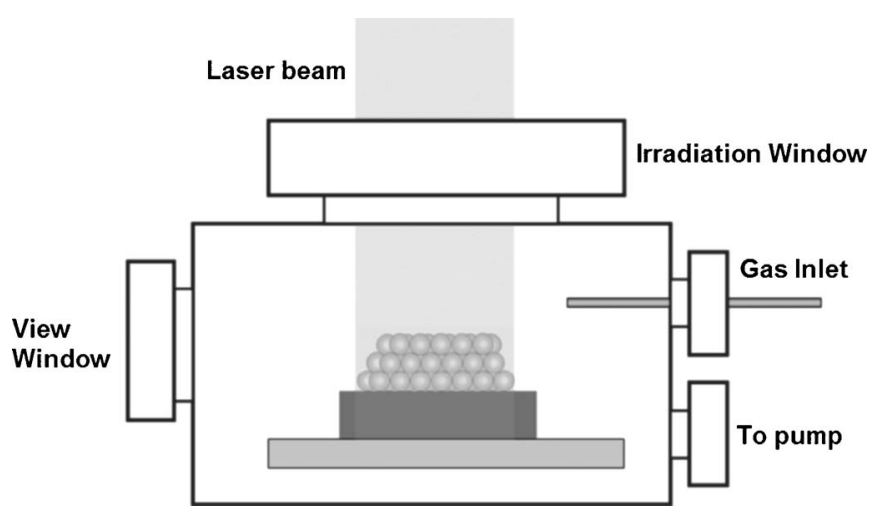

FIG. 2. Experimental setup of the LCVD system.

layers of silica particles on silicon substrates. The colloidal crystals were used as templates for LCVD of silicon.

Figure 2 shows the experimental setup of a LCVD system. A cw neodymium-doped yttrium aluminum garnet (Nd:YAG) laser (Quantronix Condor 200-IR, wavelength of $1064 \mathrm{~nm}$ ) was used to irradiate the substrates placed in a vacuum chamber. The background vacuum pressure was 1 $\times 10^{-6}$ Torr. The laser beam first irradiated the sample to heat it to $750-900{ }^{\circ} \mathrm{C}$ to remove the gas absorbed on the sample surface, before the precursor was introduced into the chamber. After the degassing process, disilane $\left(\mathrm{Si}_{2} \mathrm{H}_{6}\right)$ (Voltaix Inc., 99.998 vol \%) was introduced into the chamber with an initial pressure of 200 mTorr. During the LCVD process, the same laser power was used. The temperature field was established instantaneously and a close-chamber CVD was initiated. Disilane was used as the only precursor in the LCVD process. As the reaction progressed, hydrogen was generated in the chamber, with which the gas environment accelerated the reaction of silicon deposition. The original laser beam has a diameter of $6 \mathrm{~mm}$, in which a uniform deposition was obtained. The LCVD process lasted for 1.5-3 min, so that the deposited silicon could be infiltrated into the colloidal PBG structures with a thickness of 10-20 $\mu \mathrm{m}$. The high substrate temperature enabled not only rapid deposition but also simultaneous annealing. After removing the silica particles by HF acid (10 vol \%) etching, spherical hollow silicon-shell arrays were fabricated. The silicon infiltrated into the colloidal crystals could be amorphous or crystalline when the depositions were carried out at different substrate temperatures.

The experimental details are as follows. First, the initial precursor pressure was adjusted in a range of 200-500 mTorr. The lowest initial precursor pressure of 200 mTorr was sufficient for silicon-shell deposition in a colloidal crystal up to 20 particle layers. Higher initial pressure could not further increase the efficiency of LCVD. Hence, a pressure of 200 mTorr is considered sufficient for LCVD. Secondly, deposition duration was well controlled during the experiments. Compared with the conventional silicon CVD which takes several hours to infiltrate the structures, ${ }^{6,7}$ LCVD takes only $1-3 \mathrm{~min}$ to complete the deposition process due to the intensive laser energy. Finally, deposition results were dependent on the laser power. The silicon shells deposited under different laser powers were analyzed using Raman spectroscopy. 


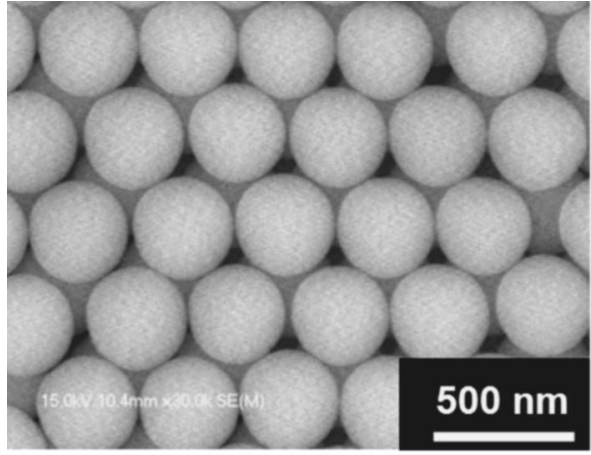

(a)

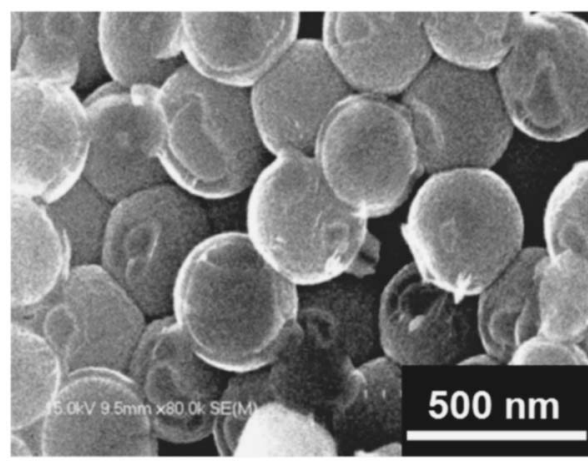

(b)

FIG. 3. SEM images of colloidal crystal and silica particles with silicon shells. (a) Top view of a colloidal crystal. (b) Cross section of silica particles deposited with silicon shells.

The PBG structures after each fabrication step were characterized using both scanning electron microscopy (SEM) and spectroscopic ellipsometry (SE), for the topographic and optical properties. The material properties of the deposited silicon were characterized using a homemade microRaman spectroscopic system, with the excitation of an argon ion laser (514 $\mathrm{nm}$ wavelength).

\section{RESULTS AND DISCUSSION}

The SEM images of the PBG structures obtained in each fabrication step are shown in Figs. 3 and 4. Figure 3(a) shows the top view of a colloidal crystal, which was used for further silicon LCVD. Figure 3(a) is the (111) surface of the fcc lattice. In the self-assembly process, the silica particles were stacked into cubic-close-packed structures. The small spacing among particles allowed diffusion of the gas reactants for infiltration. Figure 3(b) shows a cross-sectional view of the silicon shells grown on the silica particles. The cross section of silicon shells covering silica particles provides us the information about the shell thicknesses, which were used in simulating the optical properties. Generally, the entire colloidal-crystal structures were covered by the deposited silicon. The samples could be cleaved as normal silicon wafers along the crystal axis. The cross sections of the structures were exposed to enable HF etching of the silica particles. Spherical hollow silicon-shell arrays were obtained, as shown in Figs. 4(a) and 4(b).

Raman spectroscopy was performed to determine the quality of the silicon films deposited by LCVD. Two sets of samples prepared at different laser powers and deposition

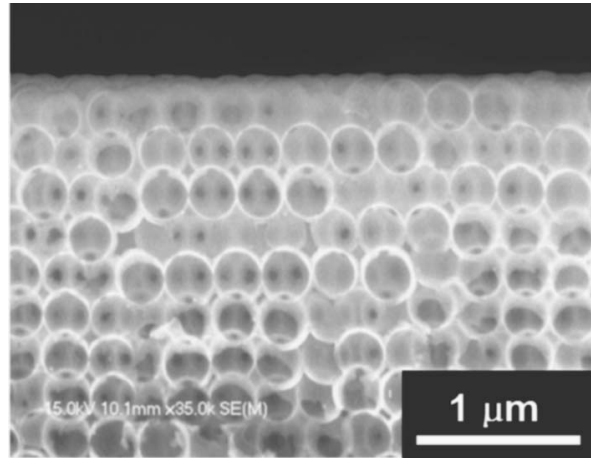

(a)

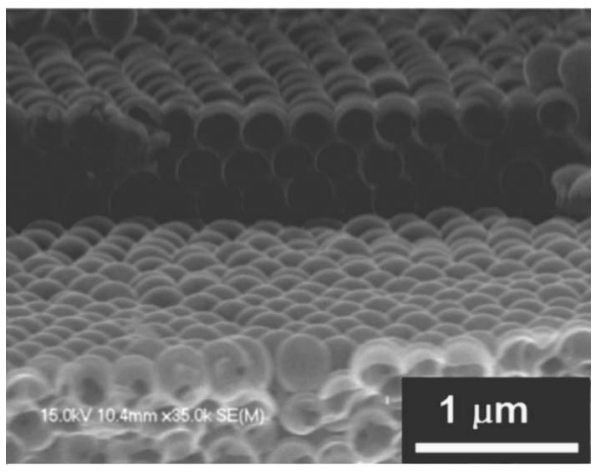

(b)

FIG. 4. SEM images of spherical silicon-shell array structures from different orientations and under different magnitudes. (a) (112) surface of the hollow spherical silicon-shell array structure. (b) Side view of the structure.

durations were used, as listed in Table I. Figure 5(a) is the Raman spectrum of a sample deposited with silicon shells at a temperature below $800{ }^{\circ} \mathrm{C}$. It is a typical Raman spectrum with amorphous silicon $(a-\mathrm{Si})$ features. The two distinct bands, at 180 and $500 \mathrm{~cm}^{-1}$, are associated with the transverse acoustic (TA) and transverse optic (TO) vibration modes, respectively. In Fig. 5(b), the Raman spectrum of another sample has a sharp peak at $524 \mathrm{~cm}^{-1}$, which indicates that the deposited silicon is crystalline $(c-\mathrm{Si})$. The band at $980-1000 \mathrm{~cm}^{-1}$ is the second order Raman peak of silicon, associated with high quality of the deposited crystalline silicon. Due to the intensive energy provided by the laser beam, the deposition process progressed rapidly with annealing occurred simultaneously.

The photonic dispersion diagrams and optical properties of the PBG structures in each fabrication step were calculated with the plane wave method and transfer-matrix method, respectively. The PBG structures were modeled as simple fcc structures with original or effective refractive indices of atoms and matrix materials. For all three PBG structures, the "atoms" were assumed to be the closely packed structures with a volume ratio of $74 \%$ against void which is

TABLE I. Comparison of different LCVD conditions and Raman spectra.

\begin{tabular}{cccccc}
\hline \hline Sample & $\begin{array}{c}\text { Laser } \\
\text { power (W) }\end{array}$ & $\begin{array}{c}\text { Substrate } \\
\text { temp }\left({ }^{\circ} \mathrm{C}\right)\end{array}$ & $\begin{array}{c}\text { Deposition } \\
\text { time (min) }\end{array}$ & $\begin{array}{c}\text { Raman } \\
\text { spectrum }\end{array}$ & Deposition \\
\hline A & 15 & 750 & 1.5 & Fig. 5(a) & $a$-Si \\
B & 20 & 870 & 3.0 & Fig. 5(b) & $c$-Si \\
\hline
\end{tabular}




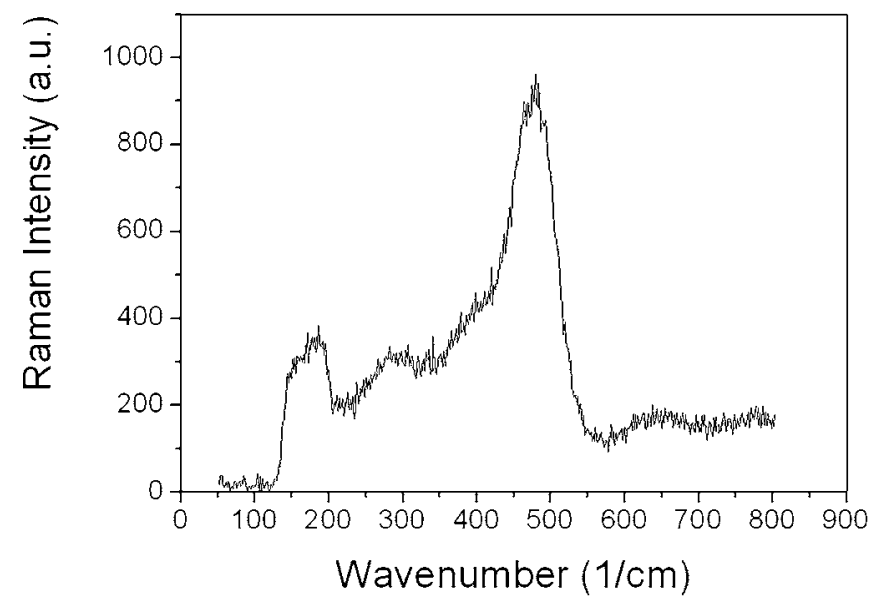

(a)

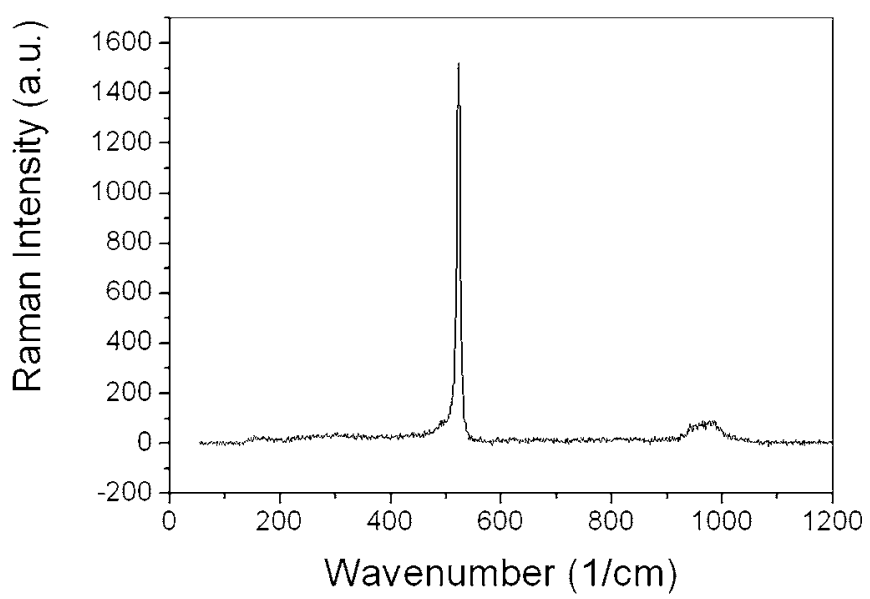

(b)

FIG. 5. Raman spectra of two samples with silicon deposited under different conditions in LCVD. (a) $a-\mathrm{Si}$ and (b) $c$-Si.

filled by air. The atoms in the models for three PBG structures with the geometric parameters are shown in Fig. 6(a). The colloidal crystals were the structures simply formed by self-assembled silica particles. The refractive indices of silica and air used to simulate these structures were 1.5 and 1, respectively. After LCVD, silicon was infiltrated into the colloidal crystal. A silica particle with a silicon shell was regarded as an individual atom with which the fcc lattice is constructed. The atom has a diameter of $345 \mathrm{~nm}$ with a $300 \mathrm{~nm}$ silica particle as the core. The parameters were taken from the cross-sectional image shown in Fig. 3(b). Because the individual atom was formed by a silica core and a silicon shell with a volume ratio of 52:48, the effective refractive index of the silica/silicon atom was 2.42 , calculated from the Bruggerman model. $^{21}$ The final structure of a hollow spherical silicon-shell array was obtained by removing the silica particles. The air-silicon "atom" has an effective refractive index of 2.25. The photonic dispersion diagrams calculated with plane wave model are shown in Figs. 6(b)-6(d), on the left. The simulated reflection spectra of the PBG structures are shown on the right as thin lines.

The reflection spectra of the PBG structures were measured using a spectroscopic ellipsometer (J. A. Woollam Co.

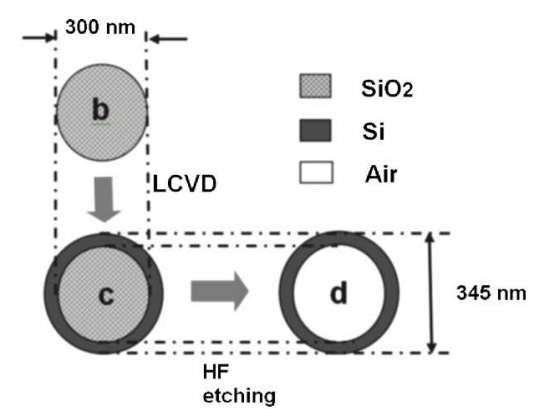

(a)

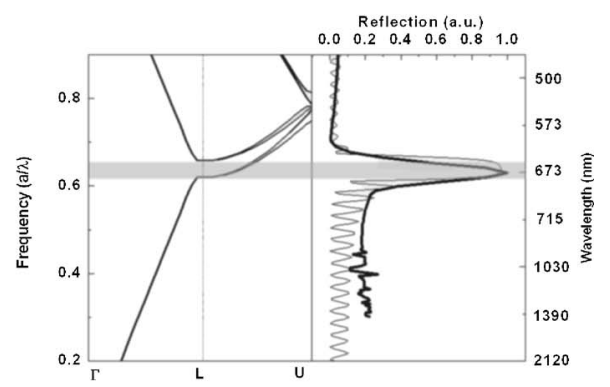

(b)

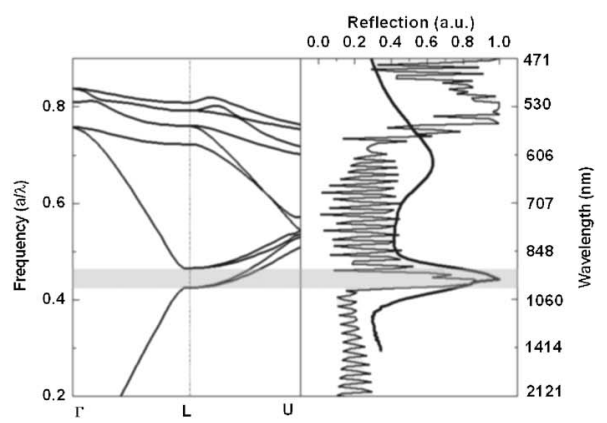

(c)

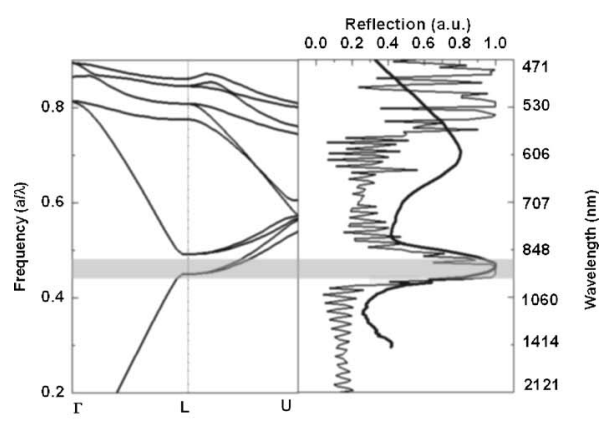

(d)

FIG. 6. (a) Schematic atom models of the PBG structures after each fabrication step. [(b)-(d)] Photonic dispersion diagrams of corresponding PBG structures (left), and the reflection spectra (right) obtained from simulation (thin curves) and measurement (thick curves), for the structures of (b) colloidal crystal, (c) silicon-shell/silica-particle structure, and (d) spherical hollow silicon-shell array structure.

M-2000). The measured reflection spectra are shown in Figs. 6(b)-6(d) on the right as thick curves. Due to the equipment constraint, the incident angle was $15^{\circ}$ to the (111) surface in reflection measurements. The reflection peak at $600 \mathrm{~nm}$ in Fig. 6(b) is the pseudophotonic band gap of the colloidal crystal. After the infiltration of silicon, the photonic band gap moved to longer wavelength and the high-energy photonic band gap entered the measurement range, because of the ex- 
istence of a material with high refraction index, as indicated in Fig. 6(c). The removal of the silica cores shifts the photonic band gap back due to the decrease of effective refractive index, as shown in Fig. 6(d). The photonic dispersion diagrams shown in Figs. 6(a)-6(c) agree with the ellipsometer measurement. The difference between simulations and measurements could be due to the slight difference of the silicon shell thickness over whole PBG structures from top to bottom. In addition, from the dispersion diagram, it is obvious that the photonic band gap width and position vary with reciprocal lattice coordinates. This indicates that, when the incident beams were irradiated from different directions, the PBGs were not the same. In this study, the $15^{\circ}$ incident angle instead of a normal incidence might also result in the difference between the simulations and measurements in obtaining the reflection spectra.

\section{CONCLUSIONS}

Spherical silicon-shell PBG structures have been fabricated using LCVD in combination with self-assembly of colloidal silica particles. The self-assembly technique yields large-area, high-quality PBG structures, which could also withstand high temperature in silicon LCVD. The laser beam used in LCVD has the advantages of high intensity, highly localized heating, and ideal thermal condition. The process developed in this study allows us to deposit crystalline silicon to infiltrate the PBG structures consisting of colloidal crystals, which led to the fabrication of 3D spherical-shell PBG structures. The spherical silicon-shell array structures could be optimized by varying precursor pressure and laser parameters. Hence, higher infiltration ratio of silicon could yield a large complete PBG. Moreover, the silicon-shell structures also promise the infiltration of quantum dots or liquid crystals for further research on functional PGB devices.

\section{ACKNOWLEDGMENTS}

This research is funded by the Air Force Office of Scientific Research (AFOSR, Grant Nos. F49620-03-1-0426 and FA9550-05-1-0453). The authors would like to thank Dr. Gernot Pomrenke (AFOSR) for his invaluable advice.

${ }^{1}$ D. V. Murphy and S. R. J. Brueck, Opt. Lett. 8, 494 (1993).

${ }^{2}$ O. Boyraz and B. Jalali, Opt. Express 12, 5269 (2004).

${ }^{3}$ L. Pavesi, L. D. Negro, C. Mazzoleni, G. Franzo, and F. Priolo, Nature (London) 408, 440 (2000)

${ }^{4}$ E. Yablonovitch, Phys. Rev. Lett. 58, 2059 (1987).

${ }^{5}$ S. John, Phys. Rev. Lett. 58, 2486 (1987).

${ }^{6}$ N. Tetreault, G. V. Freymann, M. Deubel, M. Hermatschweiler, F. PerezWillard, S. John, M. Wegener, and G. A. Ozin, Adv. Mater. (Weinheim, Ger.) 18, 457 (2005).

${ }^{7}$ G. M. Gratson, F. Garcia-Santamaria, V. Lousse, M. Xu, S. Fan, J. A. Lewis, and P. V. Braun, Adv. Mater. (Weinheim, Ger.) 18, 461 (2005).

${ }^{8}$ W. Stobe and A. Fink, J. Colloid Interface Sci. 26, 62 (1968).

${ }^{9}$ S. Wong, V. Kitaev, and G. A. Ozin, J. Am. Chem. Soc. 125, 14489 (2003).

${ }^{10}$ E. Yablonovitch and T. J. Gmitter, J. Opt. Soc. Am. A 7, 1792 (1990).

${ }^{11}$ A. Blanco et al., Nature (London) 405, 437 (2000).

${ }^{12}$ Y. A. Vlasov, X. Z. Bo, J. C. Sturm, and D. J. Norris, Nature (London) 414, 289 (2001).

${ }^{13}$ A. A. Zakhidov, R. H. Baughman, Z. Iqbal, C. Cui, I. Khayrullin, S. O. Dantas, J. Marti, and V. G. Ralchenko, Science 282, 897 (1998).

${ }^{14}$ Y. A. Vlasov, N. Yao, and D. J. Norris, Adv. Mater. (Weinheim, Ger.) 11, 165 (1999).

${ }^{15}$ M. I. Verkhovsky, A. Jasaitis, M. L. Verkhovskaya, J. E. Morgan, and M. Wikström, Nature (London) 402, 603 (1999).

${ }^{16}$ B. T. Holland, C. F. Blanford, and A. Stein, Science 281, 538 (1998).

${ }^{17}$ J. E. G. J. Wijnhoven and W. L. Vos, Science 281, 802 (1998).

${ }^{18}$ A. W. Johnson, D. J. Ehrlich, and H. R. Schlossberg, Laser-Controlled Chemical Processing of Surface (Elsevier Science, New York, 1984).

${ }^{19}$ M. Hanabusa and A. Namiki, Appl. Phys. Lett. 35, 626 (1979).

${ }^{20}$ A. Sherman, Chemical Vapor Deposition for Microelectronics: Principles, Technology, and Application (Noyes, Park Ridge, NJ, 1987).

${ }^{21}$ T. C. Choy, Effective Medium Theory: Principles and Applications (Oxford University, Oxford, 1999). 